\title{
La investigación en medicina de urgencias y emergencias
}

\section{Research in urgent and emergency medicine}

\author{
Ò. Miró' ${ }^{1}$, J. Sesma ${ }^{2}$, G. Burillo-Putze ${ }^{3}$
}

\section{RESUMEN}

La investigación sanitaria es consustancial a la excelencia clínica, hecho inherente también a la Medicina de Urgencias y Emergencias (MUE). Además, las características específicas del sistema sanitario público español y la organización de las urgencias hospitalarias y los sistemas de emergencias ofrecen un escenario probablemente único, que brinda oportunidades únicas para su análisis y estudio científico. Aunque tradicionalmente los índices de producción científica española en MUE se encontraban por debajo de los registrados por investigadores de otras especialidades médicas, así como por los urgenciólogos de otros países, desde 1995 se constata una dinámica de crecimiento continuado: es el mayor respecto a los primeros y el segundo entre nuestros colegas europeos. Además, existe mucha actividad científica de bajo nivel (comunicaciones a congresos).

Para incrementar la producción científica en MUE es preciso resolver algunos aspectos que dificultan este avance, tales como la falta de cultura científica sin duda unida al hecho de no tener especialidad reconocida (y por tanto residentes propios), la escasa formación en metodología de la investigación, la falta de reconocimiento explícito del trabajo científico, las escasas infraestructuras científicas, la dedicación prácticamente del $100 \%$ del trabajo a labores asistenciales, la poca participación en convocatorias nacionales de proyectos de investigación y la ausencia en España de una revista indexada especializada en medicina de urgencias y emergencias. Creemos además que es necesario potenciar e interrelacionar los grupos de investigación de los diferentes hospitales y sistemas de emergencias médicas, tanto localmente como entre las distintas comunidades autónomas, para que trabajen de forma coordinada y obtener así la masa crítica necesaria que permita formar una red temática de investigación cooperativa.

Palabras clave. Investigación. Medicina de urgencias. Emergencias.

\begin{abstract}
Health research is consubstantial with clinical excellence, a fact that is also inherent in urgency and emergency medicine (UEM). Besides, the specific characteristics of the Spanish health CARE system and the organisation of hospital emergency CARE offer a probably unique scenario, offering unique opportunities for their scientific analysis and study. Although the indices of Spanish scientific production in UEM were traditionally below those registered for researchers of other medical specialities, as well for UEM researchers of other countries, there has been a dynamic of continuous growth since 1995: it is the highest with respect to the former and second amongst our European colleagues. Besides, there is a great deal of low level scientific activity (papers presented to conferences).

To increase scientific production in UEM there is a need to solve some aspects that hinder this task, such as: the lack of a scientific culture, doubtless united to the lack of a recognised speciality; the limited training in research methodology; the lack of explicit recognition of scientific work; the limited scientific infrastructure; the practically $100 \%$ dedication to the work of care tasks; the scarce participation in national grants for research projects; and the absence in Spain of an indexed journal specialising in UEM. Besides, we believe that it is necessary to promote and interrelate the research groups of different hospitals and emergency medical systems, both locally and amongst the different autonomous communities, so that they work in a coordinated way and thus obtain the necessary critical mass that will enable the formation of a thematic network of cooperative research.
\end{abstract}

Key words. Research. Emergency medicine.
1. Sección de Urgencias Medicina. Área de Urgencias. Hospital Clínic. Barcelona.

2. Servicio Urgencias. Hospital de Navarra. Pamplona.

3. Servicio de Urgencias. Hospital Universitario de Canarias. Tenerife.

\author{
Correspondencia \\ Òscar Miró \\ Área de Urgencias \\ Hospital Clínic \\ Villarroel, 170 \\ 08036 Barcelona \\ E-mail: omiro@clinic.ub.es
}




\section{EL HECHO INVESTIGADOR}

Hace ya décadas que España ha superado la lapidaria frase "que inventen ellos" con la que el filósofo español Miguel de Unamuno respondió acerca de los posibles mecanismos para mejorar el avance científico español. Por similitud, esta frase derivó hacia un "que investiguen ellos", filosofía que estuvo vigente durante muchos (demasiados) años en España. No es necesario exponer aquí una extensa justificación de la necesidad de una actitud activa e intensa hacia la investigación. Baste mencionar algunos de los principales objetivos que el hecho investigador persigue para darnos cuenta que cualquier sociedad que se precie de avanzada deberá abrazar con fuerza y sin titubeos este movimiento. Así, la investigación logra nuevos conocimientos y, en el caso de la investigación biomédica, intenta que estos conocimientos redunden en beneficio del enfermo, proporcionándole una vida mejor y más larga. De forma secundaria, pero no menos importante, la investigación contribuye a mejorar el entorno productivo y el crecimiento económico de la sociedad que la promueve. Finalmente, el cultivo de la investigación permite poner en valor las mentes más preparadas y disponer del capital humano necesario para afrontar nuevos retos en un mundo que cada vez cambia a mayor velocidad y plantea más y mayores retos a resolver ${ }^{1}$. ¿Alguien puede mostrarse indiferente ante tan grandes virtudes?

\section{EL MÉDICO INVESTIGADOR}

La investigación básica o clínica es una responsabilidad de todo profesional sanitario, sea cual sea su ámbito de trabajo y su nivel de responsabilidad. Además, la investigación sanitaria es consustancial a la excelencia clínica, por lo que no se entiende ésta última sin el desarrollo de la primera. Aunque esto nos pueda parecer indiscutible en la medicina del siglo XXI, es frecuente que gestores y responsables de servicios sanitarios no piensen así y que lo único que demanden de sus profesionales sea el trabajo asistencial, que trabajen mucho y que lo hagan bien. Es más, incluso determinados responsables de contratación ven con malos ojos un currículu en el que la actividad investigadora aportada por el aspirante sea consistente y de cierta envergadura. En este ambiente, resulta fácil entender que en un área como la de la medicina de urgencias y emergencias, sin especialidad propia en la actualidad y con una elevada demanda asistencial por parte del usuario ${ }^{2,3}$, es más que probable que a los contratadores de servicios con poca visión les interese un médico de urgencias/ emergencias con un currículo exclusivamente asistencial y un bajo perfil investigador.

Pero, ¿por qué debe investigar el médico de urgencias/emergencias? Es obvio que todo médico debe basar su práctica clínica en el uso racional de la evidencia científica y, por tanto, debe participar en su búsqueda. Si no investiga él en su campo, otros lo harán ${ }^{4}$, y quizás no compartan los mismos intereses o no conozcan en profundidad las áreas peor definidas en las cuales es necesario adquirir o mejorar conocimientos. Además, investigar produce una enorme satisfacción personal y del grupo, mejora los resultados de calidad asistencial en todas sus vertientes y ayuda a evitar fenómenos de desmotivación profesional (burnout), tan frecuentes en nuestro trabajo como urgenciólogos. En el caso de las urgencias y emergencias sanitarias españolas, la falta de residentes de la especialidad es un hándicap importante. Pero tampoco debemos engañarnos y pensar que es este colectivo de médicos en formación el que hace el grueso de la investigación sustancial que se realiza en el sistema de investigación sanitaria clínica de nuestro país ${ }^{5}$. Las ideas, las preguntas clínicas, la elaboración de protocolos de investigación y la búsqueda de financiación dependen de forma directa de los médicos de plantilla de cualquier servicio. Además, la presencia de residentes de otras especialidades que rotan o hacen guardias en urgencias, que son un buen acicate clínico (en algunos casos se trata de futuros médicos de urgencias), 
también debieran serlo desde el punto de vista investigador, por lo que debiéramos transmitirles nuestra motivación y orientación hacia su formación investigadora y hacia la necesidad de dar respuestas a través de la investigación a las preguntas que plantea la clínica. Las carencias asistenciales en la Medicina de Urgencias y Emergencias, que las hay, han sido habitualmente una excusa para inhibirnos de este deber que probablemente esconde otros factores que se expondrán en este trabajo.

Además, la investigación permite de una forma menos ardua, conocer qué hacemos, cómo lo hacemos y sobre todo por qué lo hacemos de una manera determinada, con lo que se favorece nuestra actualización clínica. De esta forma, nuestra ineludible obligación de formación continuada se ve facilitada y estimulada de la mejor forma posible: con el convencimiento personal del trabajo bien hecho y la responsabilidad hacia nuestros pacientes.

Junto a las razones éticas y asistenciales, existe otra no menos importante pero probablemente infravalorada en nuestro entorno. El sistema sanitario español en general (cobertura sanitaria universal y gratuita, red de atención primaria), la organización de las urgencias hospitalarias (acceso sin cortapisas, saturación asistencial, códigos de actuación clínica específicos, unidades de observación, corta estancia, etc.) y los sistemas de emergencias (incluyendo en ellos las salas de coordinación con regulación médica, las ambulancias con médico y enfermera de forma permanente, etc.) ofrecen aspectos singulares y únicos en nuestra forma de atender a los pacientes y en la manera de gestionar esa asistencia, que probablemente difieren sustancialmente con otros países de nuestro entorno y que son diametralmente diferentes a los de EEUU, lugar del que importamos la mayoría de la evidencia científica en medicina de urgencias, sin tener en cuenta estas diferencias de organización sanitaria y sus condicionantes parasanitarios (aseguradoras, litigios, especialidad en medicina de urgencias, etc.).

\section{LA INVESTIGACIÓN EN MEDICINA DE URGENCIAS EN ESPAÑA}

La investigación en el campo de la Medicina de Urgencias y Emergencias ha sido tradicionalmente escasa en España. La primera cuantificación objetiva de esta actividad investigadora se la debemos al grupo de Montero Pérez y $\operatorname{col}^{6}$, quienes, en un estudio que abarcó 190 servicios de urgencias hospitalarios (SUH) españoles, constataron que el año 1996 sólo en un 15\% de estos SUH se habían leído tesis doctorales por sus facultativos, la mitad de ellos no había publicado ningún trabajo de investigación durante aquel año y en una tercera parte ni siquiera habían presentado alguno de sus facultativos comunicación alguna a congresos. En el mismo sentido, García Vega ${ }^{7}$ mostró como sólo un $37,2 \%$ de los médicos de urgencias que se presentaron a la oferta pública de empleo (OPE) gallega en 2003 había realizado alguna actividad científica y tan solo el $4,3 \%$ tenía el grado de doctor. Datos referentes a 2004 mostraron que en España se leyeron 48 tesis doctorales relacionadas con la Medicina de Urgencias y Emergencias ${ }^{4}$, pero tras introducir los datos de sus autores en Medline, sólo se identificaron 10 tesis doctorales como productoras de 14 trabajos de investigación difundidos en revistas indexadas mientras que el resto de tesis no generaron producción científica visible. Ciertamente, un balance muy pobre a tenor del elevado número de profesionales que desarrollan su labor en esta área de la medicina.

Probablemente, el análisis más extenso de la actividad investigadora de los urgenciólogos españoles se publicó recientemente en una serie de artículos aparecidos en la revista Emergencias ${ }^{8-10}$. Dicho análisis detalla las características de la producción científica durante un período de 30 años (entre 1975 y 2004), durante el cual se publicaron 606 artículos en revistas indexadas por Science Citation Index. Destaca que dos terceras partes de los artículos fueron publicados en castellano, la mitad correspondían a documentos originales, el factor de impacto medio fue de 
1,11 , la media de citas por documento de 3 , el índice de documentos no citados del $45 \%$ y los índices de cooperación intrahospitalaria, extrahospitalaria e internacional del $57 \%$, $19 \%$ y $4 \%$, respectivamente. Todos estos índices se encuentran por debajo de los registrados en España por investigadores de otras especialidades médicas, así como por los urgenciólogos de otros países ${ }^{9,11-13}$. Con ello, los autores concluyen que la actividad investigadora en medicina de urgencias y emergencias no sólo resultó cuantitativamente baja, sino que cualitativamente también se situó por debajo de la media.

Existen también algunos datos llamativos respecto a la actividad investigadora de los urgenciólogos españoles ${ }^{8,9}$. Por ejemplo, es destacable que la actividad investigadora en urgencias procede mayoritariamente del ámbito hospitalario, que durante el mencionado período aportó casi el 95\% de los documentos, a pesar que la medicina de urgencias implica a un alto número de profesionales y tiene una elevada actividad en el ámbito extrahospitalario y en el de la asistencia primaria. Por otro lado, la producción la lideran, por este orden, las comunidades de Cataluña, Andalucía y Madrid, que si bien suelen liderar la investigación en otros ámbitos, suelen ordenarse de manera diferente. Además, destaca que el mapa de centros con mayor actividad científica en urgencias difiere bastante del mapa de actividad científica global hospitalaria española ${ }^{11,12}$ y, así, cuatro de los diez primeros centros en productividad en medicina de urgencias (Hospital Mútua de Terrassa, Hospital Universitario Virgen de las Nieves de Granada, Hospital Universitario Reina Sofía de Córdoba y Hospital de Poniente de Almería) ocuparon durante el período 19752004 esta posición destacada a pesar que sus centros no se encontraban entre la lista de los 20 primeros centros con actividad investigadora en biomedicina y ciencias de la salud en España entre 1994 y 2002 ${ }^{11,12}$. Ello les concede un mérito añadido puesto que, en el contexto de un hospital con baja actividad investigadora, las posibilidades de los urgenciólogos de incorporarse a proyectos conjuntos de otras áreas o especialidades de su centro con las que exista transversalidad son escasas. Finalmente, la participación de la universidad es escasa (sólo en el 15\% de documentos el urgenciólogo tenía filiación universitaria) y mucho menor que en el global de la investigación española, en donde tiene una participación de alrededor del 50\% del total de documentos. En este punto, la práctica inexistencia de cátedras y/o asignaturas de urgencias en las facultades de medicina españolas contribuye a explicarlo ${ }^{14}$.

Sin embargo, esta serie de estudios ${ }^{8,9}$ también traen a la luz datos esperanzadores. Sin duda, el más destacable de ellos es la constatación de una dinámica de crecimiento continuado de la producción científica en el campo de la Medicina de Urgencias y Emergencias (Fig. 1). En efecto, la producción científica de los urgenciólogos españoles en los últimos 10 años (1995-2004) del periodo estudiado ha sido la que ha mostrado un ritmo de crecimiento mayor entre todas las especialidades médicas españolas estudiadas y la segunda entre los urgenciólogos de los países de nuestro entorno, sólo superada por la de los holandeses (Fig. 2). Ello nos hace pensar que la actividad científica de los urgenciólogos españoles parece haber entrado en una dinámica de crecimiento progresiva que, de mantenerse, ha de permitir equipararnos cuantitativamente durante la próxima década con la actividad científica que realizan nuestros colegas españoles de otras especialidades y con la de urgenciólogos de otros países. En el mismo sentido, estos "brotes verdes" también comienzan a verse en la actividad científica congresual: en el $5^{\circ}$ Congreso Mediterráneo de Medicina de Urgencias, celebrado en Valencia en septiembre de 2009, España se situó en el tercer lugar de los países con comunicaciones situadas en el "top 50" de las mejores comunicaciones presentadas, sólo por detrás de EE.UU. y Reino Unido ${ }^{15}$ (Tabla 1), si bien es conocido que en muchas ocasiones, quizás en más del $90 \%$ de los $\operatorname{casos}^{16,17}$, las comunicaciones a congresos constituyen el punto y final de un proyecto de investigación. 

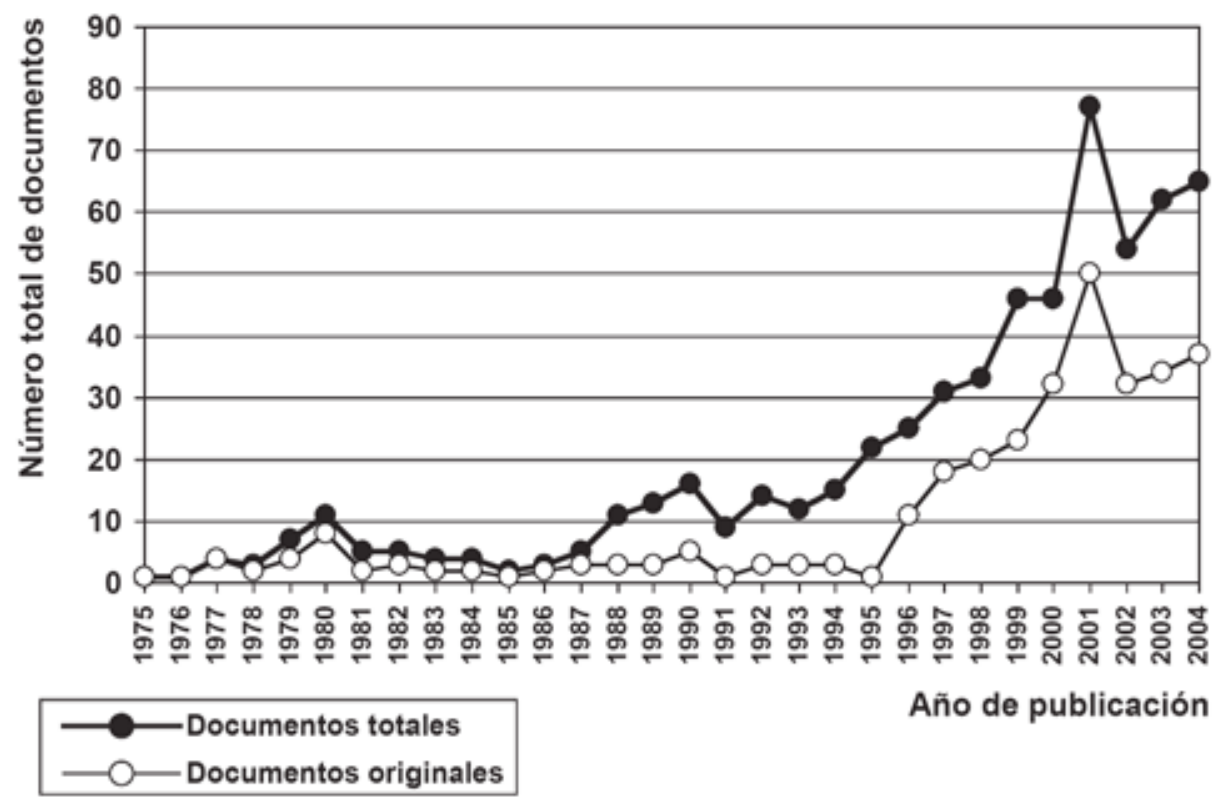

Año de publicación

Figura 1. Evolución de la producción científica indexada firmada por urgenciólogos españoles en el período comprendido entre 1975 y 2004 (tomada de Emergencias 2007; 19: 6-15 con permiso de la editorial).

Tabla 1. País de origen de las 50 mejores comunicaciones presentadas al V Congreso Mediterráneo de Medicina de Urgencias (MEMC). Valencia, Septiembre $2009^{15}$.

\begin{tabular}{|ll|}
\hline \multicolumn{1}{|c|}{ País } & N (\%) \\
\hline USA & $25(50)$ \\
\hline UK & $5(10)$ \\
\hline España & $4(8)$ \\
\hline Australia & $3(6)$ \\
\hline Israel & $3(6)$ \\
\hline Corea del Sur & $3(6)$ \\
\hline Canadá & $3(6)$ \\
\hline Irán & $2(4)$ \\
\hline Taiwan & $2(4)$ \\
\hline Italia & $1(2)$ \\
\hline Bélgica & $1(2)$ \\
\hline Suiza & $1(2)$ \\
\hline Dinamarca & $1(2)$ \\
\hline
\end{tabular}

Todos los datos anteriores deben matizarse en dos aspectos. Por un lado, si bien la cuantificación de la actividad científica puede realizarse actualmente con relativa facilidad, la calidad de las publicaciones siempre es un aspecto mucho más difícil de valorar. El número de citas que recibe un artículo en particular puede mejorar la estimación cualitativa, aunque también esto se ve influenciado por factores extrínsecos a la calidad del propio artículo, como son la visibilidad de la revista, el idioma de la misma y su factor de impacto $^{5,18}$. Por otro lado, es probable que un volumen de documentos realizados por urgenciólogos españoles no aparezca en los datos anteriores si su filiación no recoge la pertenencia a un servicio de urgencias, pero especialmente si sus trabajos no se publican en revistas indexadas. El hecho que en España no exista ninguna revista del ámbito de la Medicina de Urgencias y Emergencias indexada supone una dificul- 
tad importantísima en la proyección de la investigación que llevan a cabo este colectivo de profesionales. Baste decir que cerca de la mitad de la producción científica de los urgenciólogos se canaliza a través de EMERGENCIAS, una revista de amplia difusión, con una tirada de cerca de 10.000 ejemplares, pero que a día de hoy no se encuentra indexada. Esta publicación, no visible internacionalmente, se diferencia, con respecto a la producción visible, en una procedencia territorial diferenciada (Tabla 2) y en unas características diferenciales propias: implica un menor número de originales, es firmada por menos autores, el urgenciólogo firma con mayor frecuencia en primer lugar, existe más participación de urgenciólogos extrahospitalarios y con mayor frecuencia los urgenciólogos corresponden a servicios de urgencias independientes (autónomos respecto a otros servicios hospitalarios $)^{10}$.

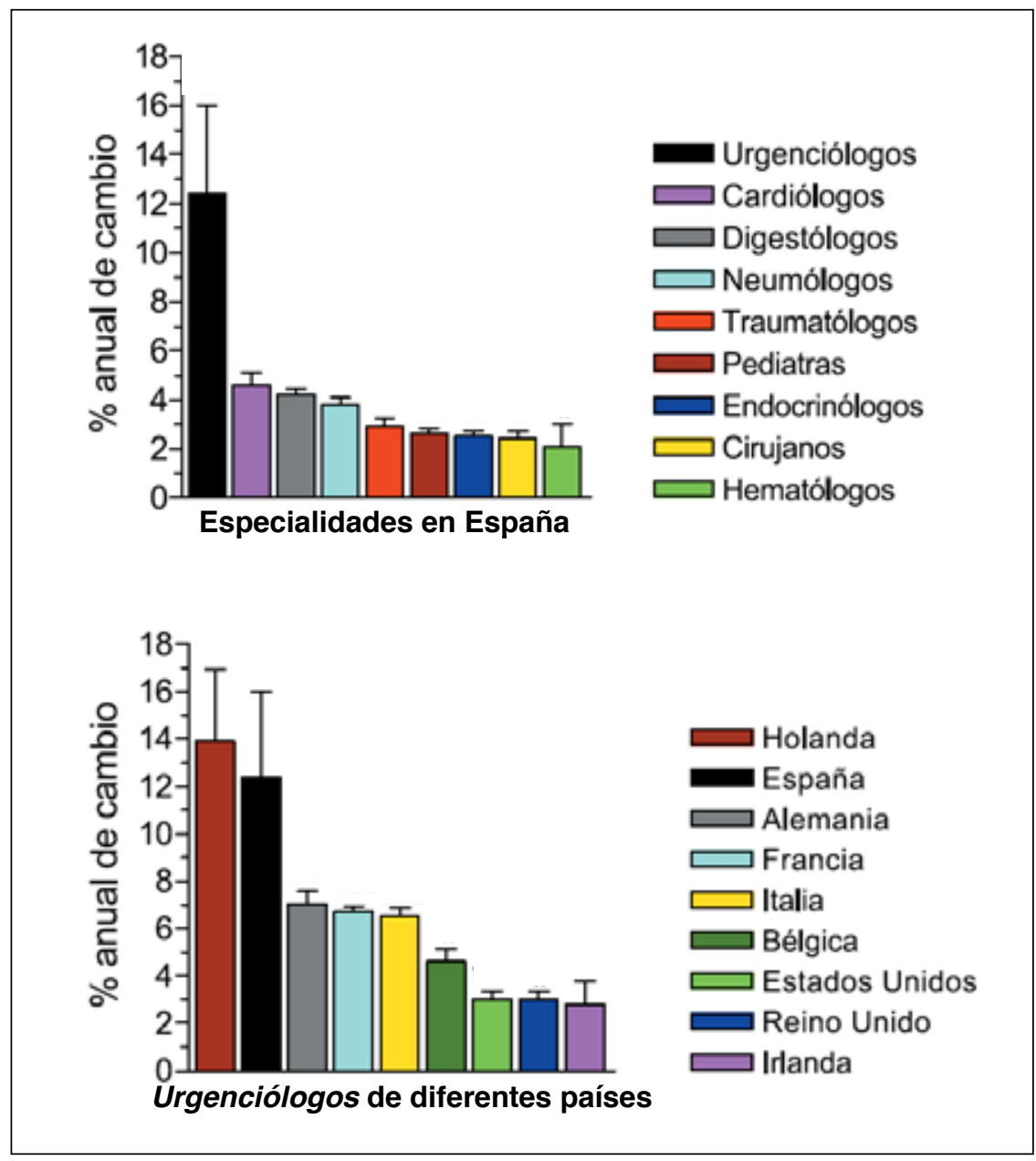

Figura 2. Comparación del crecimiento en producción científica durante el período 1995-2004 alcanzada por los urgenciólogos españoles en relación a otros compañeros de profesión (tomada de Emergencias 2007; 19: 59-64 con permiso de la editorial). 
Tabla 2. Relación de los centros que publicaron al menos 5 documentos en algunas de las categorías firmados por urgenciólogos durante el período 2000-2004, en la revista Emergencias o en revistas indexadas en el Science Citation Index (SCI).

\begin{tabular}{|c|c|c|c|}
\hline \multicolumn{2}{|l|}{ Emergencias } & \multicolumn{2}{|l|}{ Revistas del SCI } \\
\hline Hospital/SEM & $\mathbf{N}(\%)$ & Hospital/SEM & $\mathbf{N}(\%)$ \\
\hline Clínica Puerta de Hierro, Madrid & $28(13,0)$ & Hospital Clínic, Barcelona & $33(10,9)$ \\
\hline Hospital de Guipúzcoa & $8(3,7)$ & $\begin{array}{l}\text { Hospital Universitario Virgen del } \\
\text { Rocío, Sevilla }\end{array}$ & $19(6,3)$ \\
\hline Hospital Miguel Servet, Zaragoza & $8(3,7)$ & Hospital de Poniente, Almería & $15(4,9)$ \\
\hline Hospital Virgen de la Luz, Cuenca & $7(3,2)$ & $\begin{array}{l}\text { Hospital Universitario Virgen de las } \\
\text { Nieves, Granada }\end{array}$ & $14(4,6)$ \\
\hline Hospital Obispo Polanco, Teruel & $7(3,2)$ & $\begin{array}{l}\text { Hospital Universitario de Santiago de } \\
\text { Compostela }\end{array}$ & $12(3,9)$ \\
\hline Hospital de Poniente, Almería & $7(3,2)$ & Hospital Sant Pau, Barcelona & $10(3,3)$ \\
\hline $\begin{array}{l}\text { Hospital Marqués de Valdecilla, } \\
\text { Santander }\end{array}$ & $7(3,2)$ & $\begin{array}{l}\text { Hospital Universitario Ramón y Cajal, } \\
\text { Madrid }\end{array}$ & $10(3,3)$ \\
\hline $\begin{array}{l}\text { Sociedad Española de Medicina de } \\
\text { Urgencias y Emergencias (SEMES) }\end{array}$ & $6(2,8)$ & $\begin{array}{l}\text { Hospital Universitario de Canarias, } \\
\text { Tenerife }\end{array}$ & $9(3,0)$ \\
\hline 061-Madrid & $6(2,8)$ & $\begin{array}{l}\text { Hospital Germans Trias i Pujol, } \\
\text { Badalona }\end{array}$ & $9(3,0)$ \\
\hline $\begin{array}{l}\text { Hospital Universitario Reina Sofía, } \\
\text { Córdoba }\end{array}$ & $6(2,8)$ & Hospital de Basurto, Bilbao & $6(2,0)$ \\
\hline Hospital del Mar, Barcelona & $6(2,8)$ & $\begin{array}{l}\text { Hospital Clínico Universitario San } \\
\text { Carlos, Madrid }\end{array}$ & $6(2,0)$ \\
\hline Hospital Mútua de Terrassa & $5(2,3)$ & Hospital Juan Ramón Jiménez, Huelva & $6(2,0)$ \\
\hline $\begin{array}{l}\text { Hospital Universitario de San Agustín, } \\
\text { Asturias }\end{array}$ & $5(2,3)$ & Hospital Mútua de Terrassa & $6(2,0)$ \\
\hline SEM de Asturias & $5(2,3)$ & Fundación Jiménez Díaz, Madrid & $5(1,6)$ \\
\hline Hospital General de Albacete & $5(2,3)$ & Hospital Carlos Haya, Málaga & $5(1,6)$ \\
\hline $\begin{array}{l}\text { Hospital San Millán y San Pedro, } \\
\text { Logroño }\end{array}$ & $5(2,3)$ & SEM-061 de Barcelona & $5(1,6)$ \\
\hline $\begin{array}{l}\text { Resto de hospitales con menos de } 5 \\
\text { documentos }\end{array}$ & $169(58,3)$ & $\begin{array}{l}\text { Resto de hospitales con menos de } \\
5 \text { documentos }\end{array}$ & $122(40,1)$ \\
\hline
\end{tabular}




\section{FACTORES A MEJORAR EN LA INVESTIGACIÓN EN MEDICINA DE URGENCIAS Y EMERGENCIAS}

Es obvio que, para que la investigación en medicina de urgencias y emergencias dé el salto final y se equipare a la investigación que realizan el resto de profesionales médicos, es necesario que se corrijan a corto y medio plazo una serie de déficit que a día de hoy se encuentran presentes en gran parte de los centros y en un elevado número de profesionales. Los más destacables se discuten a continuación.

\section{Falta de cultura científica}

El proceso de investigación biomédica no debe acabar con la comunicación a un congreso. Éste es sólo el paso inicial, es simplemente la puesta en común de los datos preliminares o parciales del estudio en marcha y en el cual el autor recibe numerosos y valiosos comentarios. El hecho investigador necesariamente debe concluir con la publicación en forma de artículo, pues así podrá ser sometido a la crítica de la comunidad científica y ser visible por otros grupos de clínicos y/o investigadores de forma perdurable $\mathrm{y}$ rescatable. Tampoco son ajenos a este incomprensible atasco las tesis doctorales realizadas en nuestro campo. Sin duda, es responsabilidad de los profesionales más experimentados el inculcar esta cultura a las nuevas generaciones.

\section{Escasa formación en metodología de la investigación}

Es evidente que por parte de los facultativos de urgencias y emergencias, muchos de los cuales no han seguido una formación homogénea ni reglada, existen importantes lagunas en metodología, estadística, redacción, búsqueda bibliográfica o conocimiento de la lengua inglesa. Aquí debe abogarse por la realización de cursos de formación específicos por parte de las sociedades y estamentos implicados.

\section{Falta de reconocimiento explícito del trabajo científico}

Esto se da por parte de algunos responsables de servicios de urgencias/emergencias y queda patente con la mínima representación de la actividad investigadora en los baremos de méritos para el acceso a plazas y para la promoción profesional. Aunque con mayor o menor acierto, ya se ha incluido en los baremos de la carrera profesional hospitalaria, persiste este déficit en el ámbito de las emergencias. Entendemos que la línea a seguir es la de incorporar la investigación de calidad (y no sólo las comunicaciones a congresos) en los méritos profesionales de acceso y promoción interna. Determinados aspectos de investigación como las publicaciones, el nivel de la revista, el índice de citas, podrían ser el factor limitante para determinados ascensos o para ocupar puestos de responsabilidad, sobre todo aquellos ligados a la formación, a la docencia y a la calidad, tanto en el ámbito hospitalario como en el de las emergencias.

\section{Falta de infraestructuras}

Actualmente, existen carencias en apoyos logísticos de soporte y asesoramiento metodológico, estadístico, bibliográfico, carencia en infraestructuras como locales, laboratorios experimentales, animalarios, quirófanos experimentales, etc. Aquí es preciso crear sinergias con servicios de apoyo a la investigación, unidades de investigación, etc., tanto de hospitales como de universidades. Además, la implantación del grado de enfermería va a generar nuevas expectativas en este colectivo, que hemos de aprovechar. En ocasiones, además, se engloba en los servicios de emergencias las actividades de investigación con las de docencia y, más genéricamente, con las de calidad. Aunque hay puntos coincidentes entre estas tres actividades, debe organizarse la investigación de forma autónoma, para que se desarrolle de forma adecuada y no quede eclipsada por las anteriores, sin duda igualmente necesarias. 


\section{Falsa creencia de que el trabajo en urgencias/emergencias debe ser eminentemente asistencial}

Aunque la actividad asistencial debe ser el núcleo fundamental de todo profesional médico, el urgenciólogo debe tener reservado unos tiempos de su jornada laboral para dedicarse a la labor investigadora. Estos tiempos deben ser exactamente equivalentes a los que disponen sus colegas de otras especialidades, si bien puede darse la diferencia de que, mientras que éstos lo tienen de forma discontinua y discrecional a lo largo del día porque su tipo de actividad se lo permite, es posible que el urgenciólogo deba concentrárselos en determinados periodos fijos y explicitados dado que en muchos casos no es posible realizar investigación durante la jornada laboral asistencial. Por tanto, corresponde a la administración, a través de sus gestores sanitarios, proporcionar los medios, el tiempo y el dinero para investigar. Ello no es óbice para que a nivel personal uno, a menudo, deba dedicar un trabajo extra que ni está remunerado ni está contemplado en el contrato. No existe el investigador que no haya comenzado (y muchas veces continuado) su actividad científica fuera de sus horas de trabajo, si bien esta actitud inicial debe entenderla como una inversión a largo plazo de la que sin duda va a salir beneficiado. Este tiempo debe pactarse en base a resultados, de tal forma que nos comprometamos con la administración a ser eficientes en nuestra producción científica. Además, no se debe esperar a que nos den el tiempo para empezar a "hacer algo", pues suele ser más convincente presentar un bagaje investigador, aunque sea mínimo, al pactar estos objetivos.

\section{Falta de apoyo y, sobre todo, de autoestima para competir en las convocatorias nacionales de proyectos de investigación}

Esto sucede a pesar que nuestras áreas de trabajo figuran habitualmente como lí- neas prioritarias y de las facilidades que el sistema da a investigadores noveles. En este sentido, además, son especialmente asequibles las convocatorias de los servicios autonómicos de salud y de investigación, que permiten realizar estudios de poco presupuesto, fundamentales para adquirir la solvencia necesaria para las convocatorias de mayor competitividad 19-21. El fomentar la participación en esta dinámica competitiva por la obtención de recursos es indispensable para cualquier servicio de urgencias o emergencias que pretenda estar a la vanguardia de la investigación. Existen en España varias experiencias de financiación europea en proyectos transnacionales para investigación en el campo de las emergencias sanitarias, fundamentalmente ligados a la aplicación de nuevas tecnologías, al impacto sobre los ciudadanos y a la mejora de atención a las patologías emergentes. Además de los requerimientos de resultados fijados por las autoridades financiadoras, sería deseable una mayor difusión de los resultados mediante su publicación en revistas científicas ${ }^{22-24}$.

\section{Ausencia en España de una revista indexada especializada en medicina de urgencias y emergencias}

Es indudable que esto supone un hándicap importante. Ello conduce a publicar los mejores trabajos en revistas indexadas y con frecuencia extranjeras, cuya lengua vehicular es el inglés, ya que el investigador busca para su producción científica el mayor factor de impacto posible. Ello limita en ocasiones el acceso a información acerca de lo que se está investigando en este campo, ya que el urgenciólogo tiene un acceso escaso a este tipo de publicaciones. En este sentido, EMERGENCIAS, la revista de este campo con mayor difusión en España, se encuentra dando pasos firmes para conseguir esta indexación y convertirse así en una ventana atractiva desde la que divulgar los resultados de la investigación en medicina de urgencias y emergencias que se realiza en España ${ }^{25-28}$. 


\section{Falta de una red temática de investigación cooperativa}

Éste es el camino a través del cual se avanza de forma transversal y multicéntrica en los grandes campos del conocimiento en general y de la medicina en particular. Una de las posibilidades de potenciar la investigación en la Medicina de Urgencias y Emergencias sería el participar en las convocatorias del Instituto de Salud Carlos III, relativa a las urgencias y las emergencias. El conseguir una red de este tipo daría mayor consistencia a la actividad actual, aunaría esfuerzos, medios y proyec$\operatorname{tos}^{18}$. Esta red podría disponer de fondos propios para investigación y facilitaría la incorporación de grupos de investigación emergentes. Estamos convencidos de que tendría además el mismo interés para las autoridades sanitarias y científicas que otras redes ya existentes como la de atención primaria o la de salud pública ${ }^{29,30}$, pero en estos momentos nos falta masa crítica suficiente para cumplir con los requisitos mínimos para su aprobación.

\section{FUTURO DE LA INVESTIGACIÓN EN URGENCIAS/EMERGENCIAS}

Estamos convencidos que preguntas científicas e ideas originales en Medicina de Urgencias y Emergencias no faltan en el ámbito hospitalario y prehospitalario. Además, debemos ser proactivos para romper el círculo vicioso de la baja calidad en nuestras publicaciones, de que nuestra actividad científica sólo fragüe en comunicaciones a congresos y de que nuestra presencia universitaria sea escasa. Así, en el ámbito hospitalario, la cada vez más creciente codificación diagnóstica de los servicios de urgencias y la informatización de la historia clínica hacen que cada día sea más fácil acceder a la información de nuestros pacientes, en mucho menos tiempo y de forma más clara y precisa, siempre salvaguardando los principios éticos que deber regir la actividad investigadora $^{31}$. Además, el sistema de triaje, el cual se aplica de manera exclusiva en nuestro entorno de trabajo, proporcionará datos que, de no ahondar nosotros mismos en su obtención e interpretación, nadie lo hará y se perderá una oportunidad inmensa de contar con información objetiva y relevante que dé respuestas a preguntas de gran importancia referentes a aspectos de organización y flujo de pacientes en urgencias. Por su parte, la informatización desde sus inicios y las peculiaridades del modelo de Sistema de Emergencias Médicas español hacen que se disponga de un volumen ingente de información que, con el estímulo adecuado, puede dar respuesta a muchas preguntas epidemiológicas, clínicas, logísticas e incluso psicológicas, económicas y circadianas.

Quizás una de las principales asignaturas que quede pendiente sea la integración real del entorno intra y extrahospitalario en el abordaje de las preguntas de investigación ya que, de producirse, ésta permitiría general un mayor número de estudios y con un abordaje más integral desde la perspectiva de la Medicina de Urgencias y Emergencias. Ello enriquecería nuestro cuerpo doctrinal y mejoraría la atención urgente de nuestros pacientes. Algunos pasos ya se están dando en este sentido con la implantación de los códigos de activación para ciertas patologías prevalentes en las que el factor tiempo reviste especial importancia ${ }^{32}$. Esta integración sin duda debe también tener en cuenta la diferente procedencia académica de los profesionales que a día de hoy prestan asistencia urgente, tanto médicos como enfermeros y técnicos de transporte sanitario, los cuales también deben llevar a cabo los estudios específicos ${ }^{33}$ que precisen para profundizar en los conocimientos que su actividad implica y genera.

Finalmente, y como se ha mencionado anteriormente, es completamente necesario potenciar los grupos de investigación en los diferentes servicios, hospitales y sistemas de emergencias médicas de diferentes comunidades autónomas y estructurar los mecanismos para que éstos puedan trabajar de forma coordinada. Aunque existen algunos ejemplos de grupos de investigación altamente competitivos y productivos durante los últimos años, como pue- 
den ser los de insuficiencia cardiaca ${ }^{34-39}$ o toxicología $a^{40-46}$, es imprescindible que esta actitud se generalice al grueso del campo de conocimientos de nuestro cuerpo doctrinal. Esta apuesta es fundamental, ya que debemos aunar esfuerzos para no quedar descolgados de la próxima edición del Plan Nacional de I+D+I que entrará en vigor en 2012 y, en general, de la Estrategia Nacional de Ciencia y Tecnología ${ }^{47}$. Se hace imprescindible que, para ese momento, nuestra masa crítica de investigadores, de grupos de investigación consolidados y de centros de excelencia constituyan el primer nodo de calidad: una Red Temática de Investigación Cooperativa en Medicina de Urgencias y Emergencias. Sin duda, en el momento en el que se consiga, iniciaremos un período de normalidad investigadora que ya debiera haberse producido hace años de no haber sido por la ausencia de especialidad que nos viene lastrando desde hace ya demasiado tiempo.

\section{BIBLIOGRAFÍA}

1. Rozman C. Reflexiones sobre la investigación biomédica en España. Med Clin (Barc) 2003; 120: 19-23.

2. Miró Ò. El usuario y su uso de las urgencias. An Sist Sanit Navar 2009; 32: 311-316.

3. PÉrez-Ciordia I, Guillén Grima F. Urgencias hospitalarias y extrahospitalarias en Navarra. Razones que las motivan. An Sist Sanit Nav 2009; 32: 371-384.

4. Burillo-Putze G, García-Castrillo Riesgo L, Miró Andreu Ò, Montero Pérez J, Nuñez Diaz S, Tomas Vecina $\mathrm{S}$ et al. ¿Qué investiguen ellos? Emergencias 2005; 17: 107-111.

5. Jiménez-Contreras E, Torres-Salinas D, RuizPérez R, Delgado López-Cózar E. Investigación de excelencia en España: ¿protagonistas o papeles secundarios? Med Clin (Barc) 2010; 134: 76-81.

6. Montero Pérez FJ, Calderón de la Barca Gázquez JM, Jiménez Murillo L, Berlango Jiménez A, Pérez Torres I, PÉrula DE Torres L. Situación actual de los Servicios de Urgencias Hospitalarios en España (II): Actividad asistencial, docente e investigadora. Emergencias 2000; 12: 237-247.

7. García Vega FJ. Reflexiones sobre la OPE de urgencias hospitalarias en Galicia. Emergencias 2004; 16: 93-94.
8. Miró Ò, Salgado E, González-Duque A, Tomás S, Burlllo-Putze G, SÁnchez M. Producción científica de los urgenciólogos españoles durante los últimos 30 años (1975-2004). Análisis bibliométrico descriptivo. Emergencias 2007; 19: 6-15.

9. Miró Ò, Salgado E, González-Duque A, Tomás S, Burillo-Putze G, SÁnchez M. Producción científica de los urgenciólogos españoles durante los últimos 30 años (1975-2004). Análisis comparativo con la actividad de otras especialidades en España y con la de urgenciólogos de otros países. Emergencias 2007; 19: 59-64.

10. Miró Ò, González-Duque A, Cinesi C, Tomás S, PACHEco A, SÁnchez M et al. Artículos publicados en EMERGENCIAS entre 2000 y 2004: participación de los urgenciólogos y comparación con su aportación en las revistas indexadas. Emergencias 2008; 20: 308-315.

11. Camí J, Suñér-Piñol E, MÉndez-VÁsquez RI. Mapa bibliométrico de España 1994-2002: Biomedicina y Ciencias de la Salud. Med Clin (Barc) 2005; 124: 93-101.

12. Camí J, Zulueta MA, Fernández MT, Bordons M, Gómez I. Spanish scientific production in biomedicine and health sciences during the period 1990-1993 (Science Citation Index and Social Science Citation Index) and comparison to period 1986-1989. Med Clin (Barc) 1997; 109: 481-496.

13. CAmí J. Cuantificando la actividad científica en urgencias. Emergencias 2007; 19: 55-56.

14. Coll-Vinent B, Sánchez M, Nogué Bou R, Miró Ò. La enseñanza de la Medicina de Urgencias y Emergencias en las facultades de medicina: situación actual. Emergencias 2010; 22: 21-27.

15. Graham C, Gaddis G, Sabbe M, Lang E. Leading Abstracts of the Fifth Mediterranean Emergency Medicine Congress (MEMC V), Valencia, España, 14-17 September 2009. Eur J Emerg Med 2009, 16: 277-293.

16. Casal Codesido JR, García-Castrillo Riesgo L, Miró Ò. Congresos de la Sociedad Española de Medicina de Urgencias y Emergencias (y II): evolución de 20 años de actividad científica. Emergencias 2008; 20: 428-434.

17. Graham C. Emergency Medicine across the globe: improving scientific writing. Eur J Emerg Med 2009; 16: 233.

18. GRAHAM C. Impact factor, change and academic emergency medicine. Eur J Emerg Med 2009; 16: 169.

19. ANDREu AL. La medicina de urgencias en el contexto de la I+D+i en España. Emergencias 2008; 20: 297-298. 
20. Grau J, Trilla A. Algunas consideraciones bioéticas acerca de la investigación con muestras biológicas en los servicios de urgencias. Emergencias 2009; 21: 382-385.

21. Rebollo García NE, Muhedini GK, Perea-Millá E, Molina Ruano R, Ordóñez Márti-Aguilar MV. Las ayudas públicas para investigación: una puerta de entrada para investigar en urgencias y emergencias. Emergencias 2008; 20: 335-342.

22. León Miranda MD, Gómez Jiménez FJ, Martín-Castro C, Cárdenas Cruz A, Olavarría Govantes L, de LA Higuera Torres-Puchol J. Factores pronósticos de mortalidad en la parada cardiorrespiratoria extrahospitalaria. Med Clin (Barc) 2003; 120: 561-564.

23. Raya Moles JA, Martín Castro C, Gómez Jiménez FJ, CAstillo Garzón M. Acceso telefónico para sordos al Sistema de Emergencias Sanitarias 061. Med Clin (Barc) 2003; 120: 663-669.

24. Álvarez-Sabín J, Molina CA, Abilleira S, Montaner J, García Alfranca F, Jiménez Fabrega X et al. Impacto de la activación del Código Ictus en la eficacia del tratamiento trombolítico. Med Clin (Barc) 2003; 120: 47-51.

25. Miró Ò. Proyecto "EMERGER»: Hacia el reconocimiento total de EMERGENCIAS. Emergencias 2007; 19: 1-2.

26. Miró Ò, Burillo-Putze G, Pacheco A, Tomás VeciNA $S$, SÁnchez M. La nueva imagen de emergencias. Emergencias 2008; 20: 1-4.

27. Miró Ò, Burillo G, Tomás S, Sánchez M, Pacheco A. Proyecto EMERGER: primera valoración de resultados. Emergencias 2008; 20: 149-151

28. Miró Ò. Emergencias: Tras los pasos de sus hermanos mayores. Emergencias 2009; 21: $1-2$.

29. Monografía sobre la redIAPP. Redes de investigación en medicamentos. Número 7. Enero 2007. Monografia sobre la redIAPP. Disponible en: http://www.medicamentos-innovadores.org/documentos/REDES_7_Revista.pdf

30. Monografía sobre la RCESP. Redes de Investigación en Medicamentos. Número 6. Julio 2006. Disponible en: http://www.medicamentos-innovadores.org/documentos/REDES_6.pdf

31. Grau J, Trilla A. Algunas consideraciones bioéticas acerca de la investigación con muestras biológicas en los servicios de urgencias. Emergencias 2009; 21: 382-385.

32. Gómez-Angelats E, Bragulat E, Obach V, GómezChoco M, SÁnchez M, Miró Ò. Resultados alcanzados con la puesta en marcha del circuito "Código ictus" en un gran hospital: papel de urgencias y análisis de la curva de aprendizaje. Emergencias 2009; 21: 105-113.

33. Amigó TADín M. La investigación de la enfermería de urgencias en España a través de la base de datos CUIDEN (2000-2005). Emergencias 2008; 20: 299-307.

34. LloRENS P. Implicación de las nuevas guías de la Sociedad Europea de Cardiología en el tratamiento de la insuficiencia cardiaca aguda: ¿debemos cambiar nuestros esquemas en urgencias? Emergencias 2009; 21: 143-147.

35. Llorens P, Martín-SÁnchez FJ, GonzÁlez-Armengol JJ, Herrero P, Jacob J, Bella Álvarez A et al. Perfil clínico del paciente con insuficiencia cardiaca aguda atendido en los servicios de urgencias: datos preliminares del Estudio EAHFE (Epidemiology Acute Heart Failure Emergency). Emergencias 2008; 20: 154-163.

36. Miró Ò, Llorens P, Martín-SÁnchez FJ, Herrero P, PAVón J, PÉREz-Durá MJ et al. Short-term prognostic factors in elderly patients seen in emergency departments for acute heart failure. Rev Esp Cardiol 2009; 62: 757-764.

37. Llorens-Soriano P, Carbajosa-Dalmau J, Fernández-Cañadas J, Murcia-Zaragoza J, Climent-Payá $\mathrm{V}$, Laghzaou $\mathrm{F}$ et al. Experiencia clínica con levosimendán en un servicio de urgencias de un hospital de tercer nivel. Rev Esp Cardiol 2007; 60: 878-882.

38. Miró Ò, Llorens P, Martín-SÁnchez FJ, Herrero P, JAсов J, PÉrez-Durá MJ et al. Predicting the risk of re-attendance for acute heart failure patients discharged from Spanish Emergency Department Observation Units. Eur J Emerg Med 2010. (En prensa).

39. Riesgo A, Herrero P, Llorens P, Jacob J, MartínSÁnchez FJ, Bragulat E et al. Influencia del sexo del paciente en la forma de presentación y en el manejo de la insuficiencia cardiaca aguda en los servicios de urgencias españoles. Med Clin (Barc) 2010. (En prensa).

40. Burillo-Putze G, Borreguero León JM, Vallbona Afonso E, De Vera González AM, Fernández RoDRíGuez JF, GARcía Dopico JA et al. Consumo de cocaína y su relación con patología cardiaca y traumática atendida en un servicio de urgencias. Emergencias 2008; 20: 380-384.

41. Burillo Putze G, Munné Mas P, Dueñas laita A, Trujillo Martín MM, Jiménez Sosa A, Adrián MARTín MJ et al. Intoxicaciones agudas: perfil epidemiológico y clínico, y análisis de las técnicas de descontaminación digestiva utilizadas en los servicios de urgencias españoles en el año 2006 -Estudio HISPATOX-. Emergencias 2008; 20: 15-26. 
42. Burillo Putze G, Pinillos Echeverría MA, Jiménez Lozano MA, Bajo Bajo A, Avilés Amat J, Berruete Cilveti M et al. Organización y disponibilidad de recursos para la asistencia toxicológica en los servicios de urgencias de los hospitales españoles. Emergencias 2006; 18: 219-228.

43. Burillo-Putze G, López B, León JM, SÁnchez MS, GonZÁlez MG, RodriguEz AD et al. Undisclosed cocaine use and chest pain in emergency departments of Spain. Scand J Trauma Resusc Emerg Med 2009; 17: 11.

44. Galicia M, Nogué S, Sanjurjo E, Miró Ò. Evolución de las consultas urgentes relacionadas con el consumo de cocaína durante el periodo 2002-2007. Emergencias 2008; 20: 385390 .
45. Burillo-Putze G, Borreguero León JM, García Dopico JA, Fernández Rodríguez JF, Pérez CARrILlo MA, Jorge Pérez MJ et al. Incidence and impact of undisclosed cocaine use in emergency department chest pain and trauma patients. Int J Emerg Med 2008; 1: 169-172.

46. Amigó Tadín M, Nogué Xarau S, Gómez López E, Sanjurjo Golpe E, Sánchez Sánchez M, PuigurIGUER FERRANDO J. Medida de la calidad asistencial que se ofrece a los pacientes con intoxicaciones agudas en el Servicio de Urgencias. Emergencias 2006; 18: 7-16.

47. Comisión Interministerial de Ciencia y Tecnología. Estragia Nacional de Ciencia y Tecnología. Disponibles en: http://www.plannacionalidi.es/plan-idi-public/documentos/ ENCYT.pdf 
